\title{
Escala de madurez gonadal del jurel Trachurus murphyi Nichols 1920
}

\author{
Scale of gonad maturity stages of Jack mackerel Trachurus murphyi Nichols 1920
}

\author{
Javier Sánchez, Ángel Perea, Betsy Buitrón y Liz Romero
}

Instituto del Mar del Perú, esquina Gamarra y General Valle S/N Chucuito, Callao, Perú.

Email Javier Sánchez: jsanchez@imarpe.gob.pe
Citación:

Sánchez J., A. Perea, B. Buitrón \& L. Romero. 2013. Escala de madurez gonadal del jurel Trachurus murphyi Nichols 1920. En: Eds. Csirke J., R. Guevara-Carrasco \& M. Espino. Ecología, pesquería y conservación del jure (Trachurus murphyi) en el Perú. Rev. peru. biol. número especial 20(1): 035 - 044 (Septiembre 2013)

\section{Resumen}

El presente trabajo describe la escala de madurez gonadal macroscópica del jurel Trachurus murphyi Nichols 1920, validada con estudios microscópicos y en uso en Perú. La escala consta de seis estadios de maduración para hembras y machos, que van desde el estadio 0 (virginal) al estadio V (recuperación en hembras, posexpulsante en machos). Para el estudio, se contó con 350 ejemplares provenientes del plan de seguimiento de la pesquería pelágica y de cruceros de evaluación de recursos pelágicos; dichos ejemplares fueron colectados desde el 2006 al 2009. La escala cataloga a los individuos virginales en el denominado estadio 0 , lo cual permite la separación práctica entre juveniles (estadio 0 ) y adultos (estadios I a V). Se discute las bondades de la escala validada para el jurel $T$. murphyi, la cual se compara con otras escalas de madurez propuestas para especies de la misma familia. El análisis de las características microscópicas de los ovarios confirman la tipificación de desove parcial ya descrita por otros autores.

Palabras claves: jurel, Trachurus murphyi, madurez gonadal, reproducción, estadios de madurez.

\section{Abstract}

This paper describes the macroscopic scale of gonad maturity stages of Jack mackerel Trachurus murphy Nichols 1920, validated by microscopic studies, and being used in Peru,. A scale of six maturity stages are identified for females and males, ranging from stage 0 (virgin) to stage $\mathrm{V}$ (recovery in females, post-spermiation in males). The gonads of 350 specimens were analyzed. These specimens were collected as part of a pelagic fishery monitoring programme and during pelagic stock assessment surveys between 2006 and 2009. Virgin individuals are classified as stage 0 (virgin), which draws the distinction between (non-reproductive) juveniles and (reproductive) adults in stages I to V. The advantages of using the validated stages of gonad development of Jack mackerel T. murphyi are discussed and are compared to other scales of maturity stages proposed for other species in the same family. The analysis of the microscopic characteristics of the ovaries confirms that Jack mackerel is a batch spawner, which has already been described by other authors.

Keywords: Jack mackerel, Trachurus murphyi, gonadal maturity, reproduction, maturity stages.

\section{Introducción}

El estudio de la reproducción de los stocks de peces es un elemento importante en el desarrollo de modelos de evaluación y el manejo de los recursos, proporcionando variables como las tallas de primera madurez y el potencial reproductivo.

En la mayoría de los casos, la determinación de la condición reproductiva de la población a través de una escala de madurez basada en la morfología gonadal externa emplea criterios que no permiten identificar a todas las hembras en desove (GluyasMillán 1994). Sin embargo, el uso de este método permite analizar un gran número de individuos con relativamente poco esfuerzo.

Por otro lado, la elaboración de escalas deben basarse en criterios que permitan la identificación de los diferentes estados de maduración para proporcionar el mínimo error posible (Gonçalves et al. 2004), lo cual puede obtenerse por el análisis histológico, que en la actualidad es fácil y rápido de aplicar, resultando en la creación de escalas
Publicado online: $\quad 11 / 10 / 2013$ Publicado impreso: $15 / 10 / 2013$ 
Tabla 1. Muestras de gónadas de jurel Trachurus murphyi utilizadas para la elaboración de la escala de madurez gonadal validada.

Table 1. Gonad samples of Jack mackerel Trachurus murphyi used for preparing the scale of gonad maturity stages being validated.

\begin{tabular}{cccc}
\hline Procedencia & Sexo & $\begin{array}{c}\text { Rango de } \\
\text { talla (cm) }\end{array}$ & $\begin{array}{c}\mathbf{N}^{\circ} \text {. } \\
\text { ejemplares }\end{array}$ \\
\hline Seguimiento 2006 & Hembra & $23-49$ & 46 \\
Seguimiento 2006 & Macho & $23-52$ & 28 \\
Seguimiento 2008 & Hembra & $22-54$ & 73 \\
Seguimiento 2008 & Macho & $26-43$ & 46 \\
Seguimiento 2009 & Hembra & $33-43$ & 9 \\
Seguimiento 2009 & Macho & $40-41$ & 2 \\
Crucero 2008 (Nov-Dic) & Hembra & 22 & 4 \\
Crucero 2008 (Nov-Dic) & Macho & $22-23$ & 6 \\
Crucero 2011 (Oct-Dic) & Hembra & $27-37$ & 61 \\
Crucero 2011 (Oct-Dic) & Macho & $28-38$ & 75 \\
\hline Total & & $22-54$ & 350 \\
\hline
\end{tabular}

basadas en criterios microscópicos (Pérez \& Figueiredo 1992, Costa \& Borges 1996, Costa 2001).

Como señalan Ferreri et al. (2009), es conveniente considerar las causas de discrepancias entre las observaciones macroscópicas e histológicas, incluidas la subjetividad del operador en la catalogación, distinción clara entre las descripciones de los estadios de madurez, muestreo durante el día y el tiempo transcurrido desde la captura hasta la catalogación de las gónadas. Si contamos con una escala de madurez bien elaborada, con estadios bien definidos (Hunter \& Macewicz 2003, Gonçalves et al. 2004, Gerritsen \& McGrath 2006), esta podría ser utilizada en el trabajo de rutina y dejar el trabajo histológico solamente para los casos en que sea estrictamente necesario.

Desde que se iniciaron los estudios sobre los aspectos reproductivos de jurel Trachurus murphyi en el Perú hace más de 40 ańos, se ha venido utilizando la escala adaptada de madurez gonadal, descrita por Johansen (1924) que consta de 8 estadios y que fue descrita para el arenque (Clupea harengus). También ha sido adaptada y aplicada al jurel T. murphyi la escala general de 5 estadios para reproductores parciales sugerida por Holden \& Raitt (1975) (Aracena et al. 1998, Cubillos 2003). Posteriormente, Andrianov (1987) propuso una escala de madurez gonadal para el jurel, de 7 estadios, donde el estadio maduro está subdividido en tres etapas de desarrollo. Dioses et al. (1989) describieron las características citológicas del desarrollo ovocitario del jurel, pero no incluyeron la descripción macroscópica de las gónadas, y no elaboraron una escala de madurez.

La manera y los objetivos de describir escalas macroscópicas validadas en peces quedaron documentados en la reunión mundial realizada en Bergen, Noruega, en septiembre de 2001, con participación de investigadores de varios países que incluyó sudamericanos como Argentina, Chile y Perú. En esta reunión se elaboró un documento (Kjesbu et al. 2003) que entre otras recomendaciones, sugiere la elaboración de escalas macroscópicas que deberían de contar con sustento histológico y criterios que simplifiquen la identificación de los estadios de madurez.

En el presente trabajo describimos las características de una escala de madurez gonadal de seis estadios para el jurel T. murphyi, tanto para hembras como para machos. Cada estadio es descrito a nivel microscópico y macroscópico, lo cual facilita la identificación macroscópica de cada estadio de madurez gonadal.

\section{Material y métodos}

Se analizaron en total 350 individuos de jurel T. murphyi, 204 gónadas (76 testículos y 128 ovarios) colectados entre enero 2006 y diciembre 2009 de los muestreos del programa de seguimiento de la pesquería pelágica; y 146 individuos (81 testículos y 65 ovarios) de los cruceros hidroacústicos realizados entre el 2008 y 2011 (Tabla 1).

Las gónadas frescas fueron rotuladas, registrando sus características macroscópicas, tomando fotografías digitales de cada una. Adicionalmente, como en trabajos similares (Gonçalves et al. 2004, Ferreri et al. 2009), fueron anotados aspectos complementarios como grado de vascularización, turgencia, textura y en algunos casos tipos de ovocitos visibles a simple vista. En el caso particular de los machos, se anotó la turgencia testicular y el semen fluyente.

Las gónadas fueron procesadas histológicamente usando la técnica de infiltración con parafina (Humason 1979). Con el uso del micrótomo de rotación, se realizaron cortes de $7 \mu \mathrm{m}$ para ovarios y $5 \mu \mathrm{m}$ para testículos. La diferencia en el espesor de los cortes de machos y hembras toma en cuenta las diferencias en la estructura ovocitaria y espermatogénica de las gónadas. Las células sexuales masculinas son de menor tamańo que las células sexuales femeninas, y por lo tanto necesitan un corte más fino para evitar su aglomeración y poder observarlas mejor.

Las secciones histológicas obtenidas fueron coloreadas con Hematoxilina-Eosina contrastante y analizadas en un microscopio compuesto. Las secciones histológicas fueron observadas en detalle y los distintos tipos de ovocitos fueron clasificados en función del número y distribución de nucléolos, grado de vitelogénesis y gotas lipídicas y ; además se determinó la presencia o no de folículos post-ovulatorios, así como ovocitos en estado de atresia. Las descripciones se basaron en las realizadas por Hunter y Goldberg (1980) y Hunter y Macewicz (1985). En el caso de los machos se identificó la histoarquitectura testicular y el desarrollo espermatogénico en los túbulos seminíferos (Abascal et al. 2004, Dadzie \& Abou-Seedo 2004), lo cual nos permitió diferenciar los estadios de maduración gonadal, teniendo en cuenta que el proceso de espermatogénesis es dinámico y algunos estadios de maduración podrían confundirse con otros.

Tabla 2. Estadios de madurez gonadal de hembras y machos de jurel Trachurus murphyi.

Table 2. Gonadal maturity stages of females and males of Jack mackerel Trachurus murphyi.

\begin{tabular}{ccc}
\hline Estadio & Hembra & Macho \\
\hline Estadio 0 & Virginal & Virginal \\
Estadio I & Reposo & Reposo \\
Estadio II & En maduración & En maduración \\
Estadio III & Maduro & Maduro \\
Estadio IV & Desovante & Expulsante \\
Estadio V & Recuperación & Posexpulsante \\
\hline
\end{tabular}




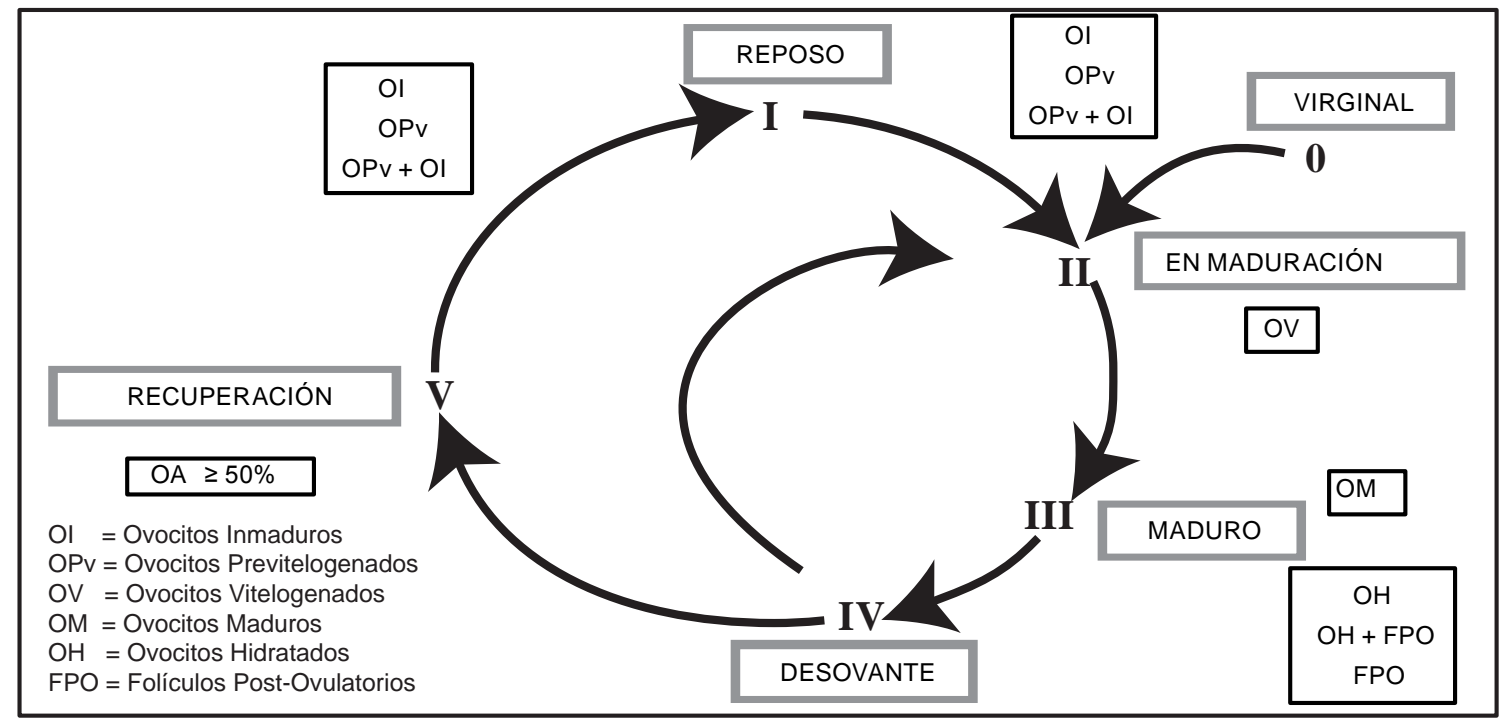

Figura 1. Ciclo de madurez gonadal de hembras de jurel Trachurus murphyi. OI: Ovocito inmaduro, OPv: Ovocito pre-vitelogenado, OV: Ovocito vitelogenado, OM: Ovocito maduro, OH: Ovocito hidratado, OA: Ovocito atrésico, FPO: Folículo post-ovulatorio.

Figure 1. Gonad maturity cycle of female Jack mackerel Trachurus murphyi. OI: immature oocyte, OPV: Pre-vitellogenic oocyte, OV: Vitellogenic oocyte, OM: Mature oocyte, OH: Hydrated oocyte, OA: Atretic oocyte, FPO: Post-ovullatory follicle.

Para cada gónada se asociaron las características histológicas y macroscópicas, lo cual permitió determinar estadios de desarrollo gonadal para ambos sexos y validar una escala de madurez gonadal macroscópica para machos y hembras.

\section{Resultados}

De acuerdo a las características macroscópicas, validadas mediante observaciones microscópicas, la escala macroscópica de madurez gonadal para hembras y machos consta de seis estadios (Tabla 2).

En la Figura 1, se ilustra el ciclo de madurez gonadal para hembras determinado mediante observaciones microscópicas, donde se observa cada estadio de madurez gonadal con los ovocitos que los caracterizan.

\section{Escala de madurez gonadal de hembras:}

\section{Virginal (0)}

Este estadio agrupa especímenes hembras que nunca maduraron gonadalmente.

Características macroscópicas: El ovario es relativamente pequeño y poco denso al tacto. Presenta un color desde anaranjado pálido o translúcido hasta anaranjado claro con algunas zonas rojizas. Es de aspecto homogéneo y poco turgente. Presenta poco desarrollo vascular (Figs. 2A y 2B).

Características microscópicas: Se observa la presencia de ovocitos inmaduros, pre-vitelogenados o ambos. Se pueden observar ovogonias en las paredes de las lamelas (Fig. 2C).

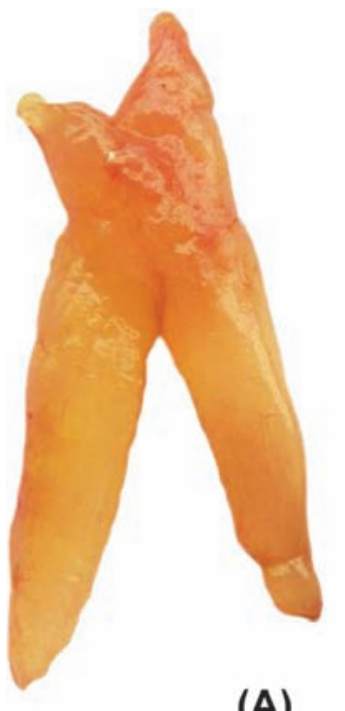

(A)

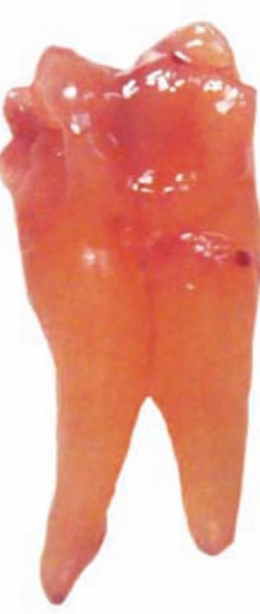

(B)

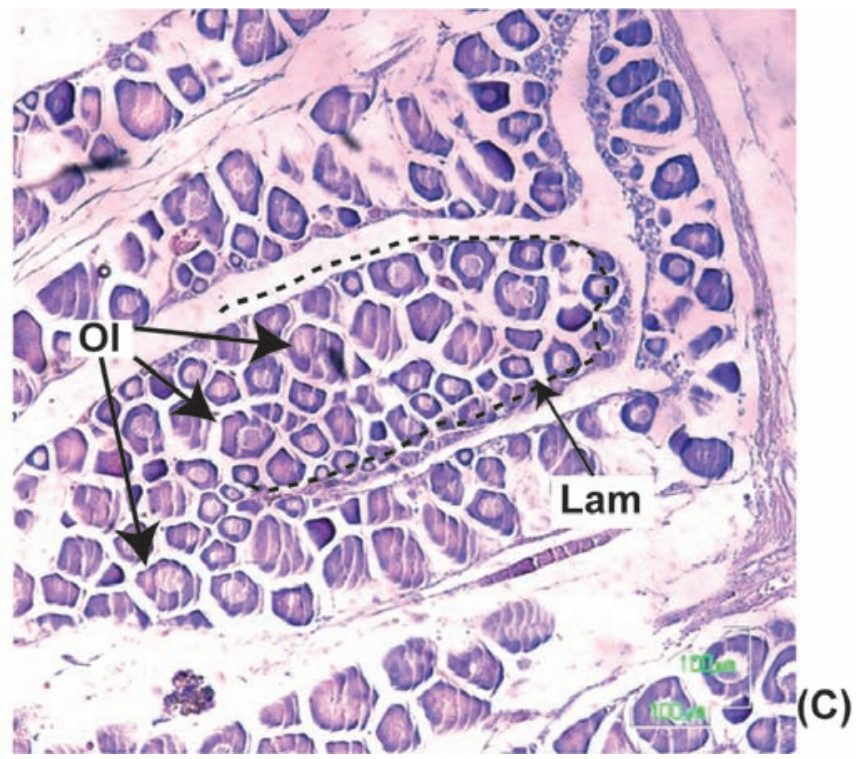

Figura 2. A y B) Fotografías de ovarios de ejemplares de $25 \mathrm{~cm}$ y $24 \mathrm{~cm}$ de longitud total y C) Microfotografía (40X) de ovario de jurel Trachurus murphyi en estadio 0 (virginal). Ol: Ovocitos inmaduros, Lam: lamelas.

Figure 2. A and B) Photographs of an ovary from individuals of $25 \mathrm{~cm}$ and $24 \mathrm{~cm}$ total length (TL) and C) Photomicrograph (40X) of an ovary of Jack mackerel Trachurus murphyi in stage 0 (virgin). OI: Immature oocytes, Lam: lamellae. 


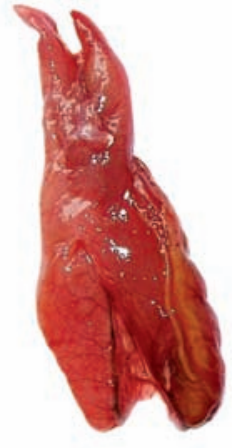

(A)

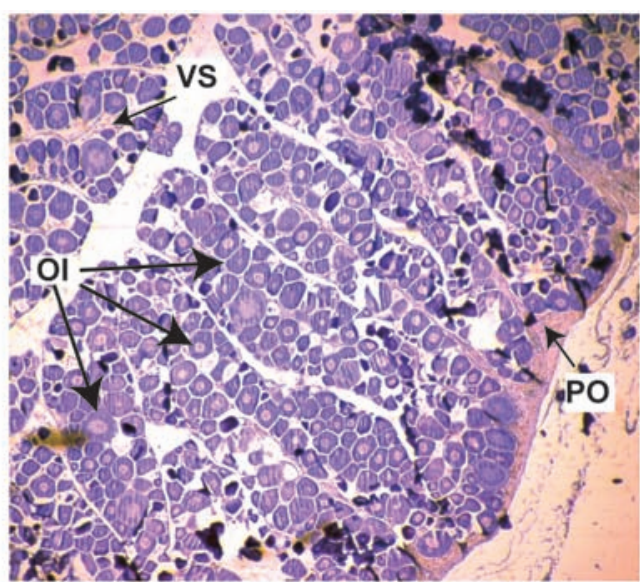

(B)

Figura 3. A) Fotografía del ovario de un ejemplar de $31 \mathrm{~cm}$ de longitud total y B) Microfotografía (40X) de ovario de jurel Trachurus murphyi en estadio I (reposo). OI: Ovocito inmaduro, VS: Vaso sanguíneo, PO: Pared del ovario.

Figure 3. A) Photograph of an ovary from an individual of $31 \mathrm{~cm}$ TL and B) Photomicrograph (40X) of an ovary of Jack mackerel Trachurus murphyi in stage I (resting). OI: Immature oocyte, VS: Blood vessel, PO: Ovary wall.

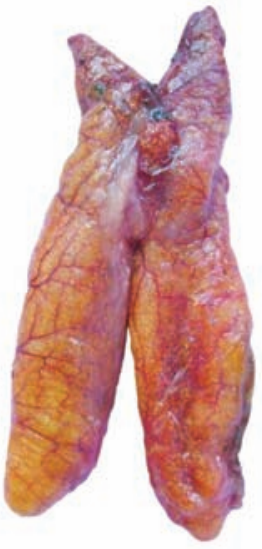

(A)

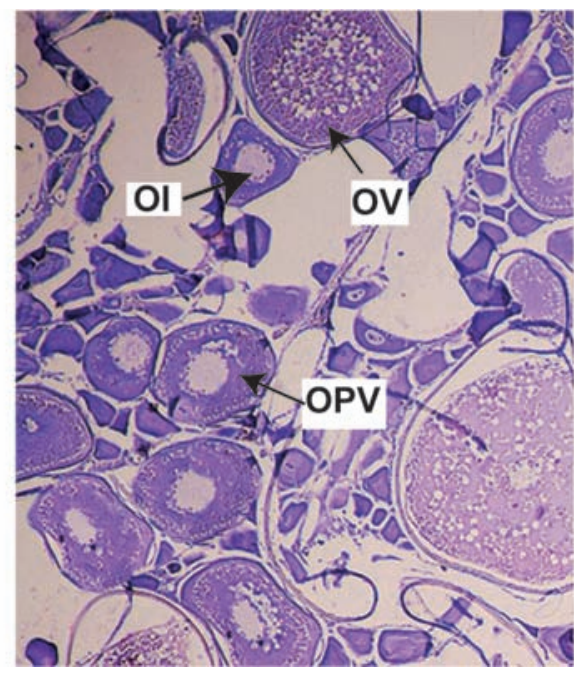

(B)

Figura 4. A) Fotografía de ovario de ejemplar de $34 \mathrm{~cm}$ de longitud total y B) Microfotografía (100X) de ovario de jurel Trachurus murphyi en estadio II (en maduración). OI: Ovocito inmaduro; OPV: Ovocito pre-vitelogenado; OV: Ovocito vitelogenado.

Figure 4. A) Photograph of an ovary from an individual of $34 \mathrm{~cm}$ TL and B) Photomicrograph (100X) of an ovary of Jack mackerel Trachurus murphyi in stage II (maturing). OI: Immature oocyte; OPV: Pre-vitellogenic oocyte; OV: Vitellogenic Oocyte.

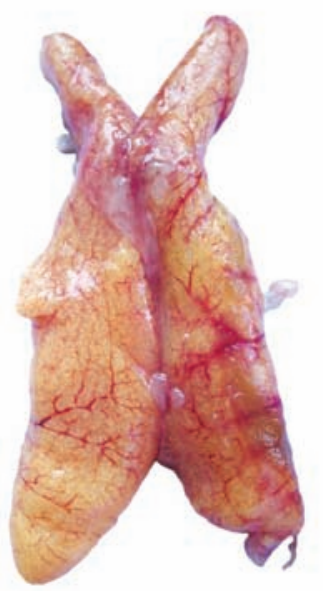

(A)

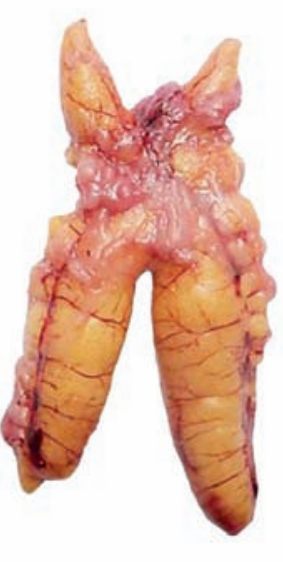

(B)

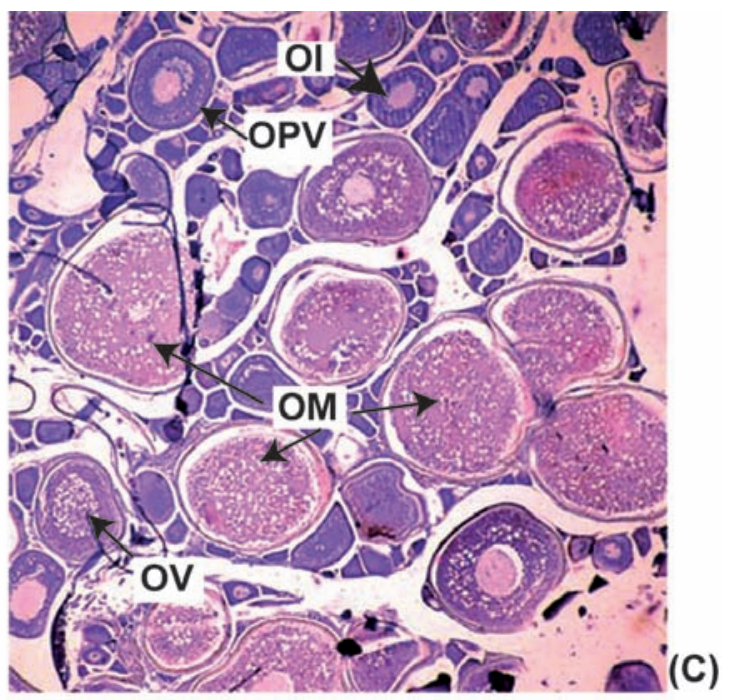

\section{(C)}

Figura 5. A y B) Fotografías de ovarios ejemplares de $34 \mathrm{~cm}$ y $36 \mathrm{~cm}$ de longitud total y C) Microfotografía (100X) de ovario de jurel Trachurus murphyi en estadio III (maduro). OI: Ovocito inmaduro, OPV: Ovocito previtelogenado, OV: Ovocito vitelogenado, OM: Ovocito maduro.

Figure 5. A and B) Photographs of ovaries from individuals of $34 \mathrm{~cm}$ and $36 \mathrm{~cm}$ TL and C) Photomicrograph (100X) of an ovary of Jack mackerel Trachurus murphyi in stage III (mature). OI: Immature oocyte, IPO: Previtellogenic oocyte; OV: Vitellogenic oocyte, OM: Mature oocyte. 


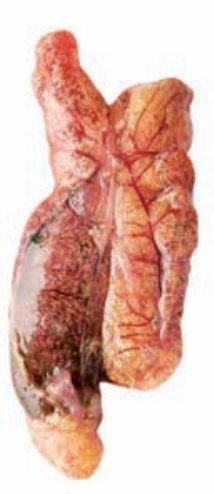

(A)

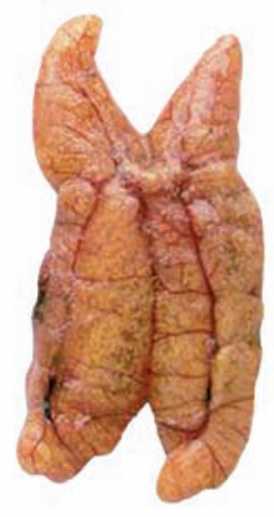

(B)

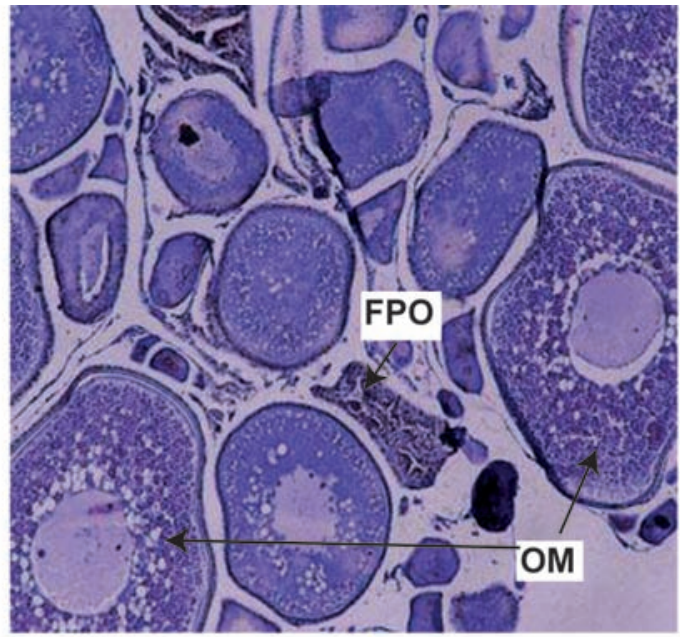

(C)

Figura 6. A y B) Fotografías de ovarios de ejemplares de $35 \mathrm{~cm}$ y de $36 \mathrm{~cm}$ de longitud total y C) Microfotografía (100X) de ovario de jurel Trachurus murphyi en estadio IV (desovante), OM: Ovocito maduro, FPO: Folículo post-ovulatorio.

Figure 6. A and B) Photographs of ovaries from individuals of $35 \mathrm{~cm}$ and $36 \mathrm{~cm}$ TL and C) Photomicrograph (100X) of an ovary of Jack mackerel Trachurus murphyi in stage IV (spawning). OM: Mature oocyte; FPO: Post-ovulatory follicle.

\section{Reposo (I)}

La identificación de este estadio es sumamente importante porque se trata de individuos considerados como adultos, pero son reproductivamente inactivos.

Características macroscópicas: Los ovarios son lisos de color naranja y medianamente turgentes, no son traslúcidos como los virginales. Los ovocitos no son visibles a simple vista, la pared ovárica es engrosada y los vasos sanguíneos están desarrollados (Figura 3A).

Características microscópicas: Desde el punto de vista ovocitario, este estadio es igual al virginal. Sin embargo, lo diferencia, principalmente, la pared gruesa del ovario y la mayor cantidad de vasos sanguíneos. En algunos casos, se observan algunos ovocitos atrésicos (Fig. 3B).

\section{En maduración (II)}

Características macroscópicas: Los ovarios son de color naranja claro. Pueden observarse zonas rosadas o rojizas dependiendo del grado de maduración en el que se encuentren (dichas zonas contienen gran cantidad de ovocitos inmaduros y previtelogenados). Son ovarios poco densos al tacto. Se pueden observar ovocitos pequeños a simple vista. Se identifica con claridad el vaso sanguíneo principal en la cara ventral del ovario (Fig. 4A).

Características microscópicas: En este estadio se observan ovocitos inmaduros, previtelogenados y ovocitos vitelogenados. (Fig. 4B).

\section{Maduro (III)}

Características macroscópicas: Los ovarios presentan diversas tonalidades de naranja, en algunos casos con zonas sanguinolentas producto de la marcada presencia de vasos sanguíneos. Son ovarios turgentes. Como en el caso del estadio en maduración, se pueden observar ovocitos a simple vista, siendo éstos de mayor tamaño (Figs. 5A y 5B).

Características microscópicas: En este estadio se observan ovocitos inmaduros, previtelogenados, vitelogenados y ovocitos

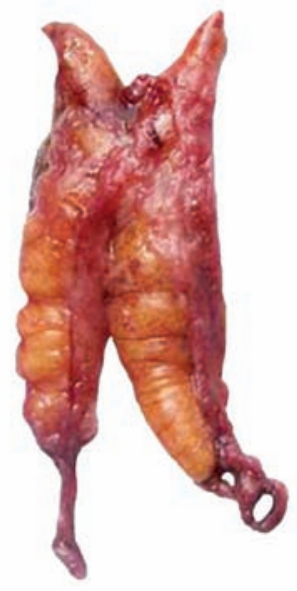

(A)

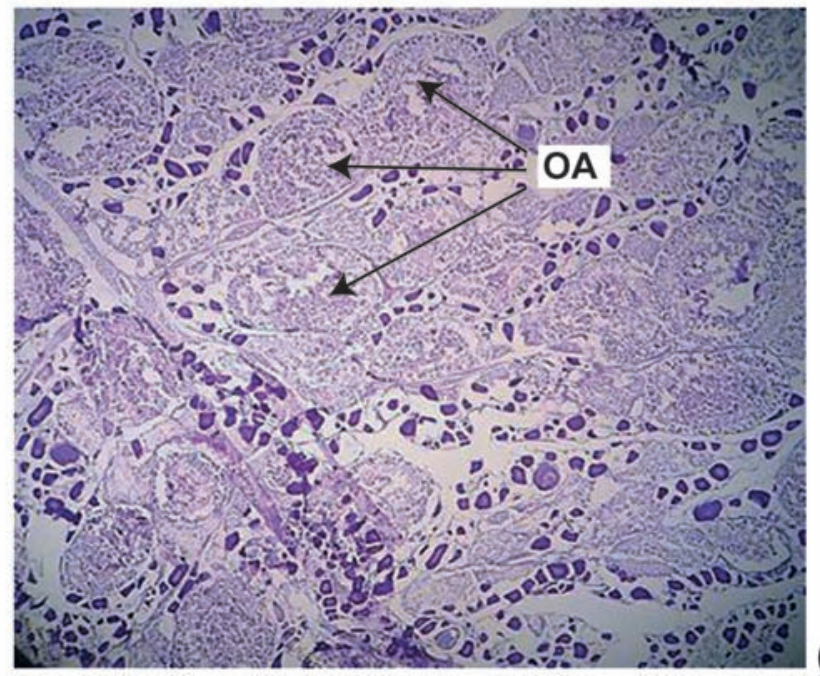

(B)

Figura 7. A) Fotografía de ovario de ejemplar de $35 \mathrm{~cm}$ de longitud total y B) Microfotografía (40X) de ovario de jurel Trachurus murphyi en estadio V (recuperación). OA: Ovocito atrésico.

Figure 7. A) Photograph of an ovary of an individual of $35 \mathrm{~cm}$ TL and B) Photomicrograph (40X) of an ovary of Jack mackerel Trachurus murphyi stage $\mathrm{V}$ (recovery). OA: Atresic oocyte. 
maduros. En algunos casos, es posible identificar ovocitos con núcleo migratorio. Igualmente, es posible identificar ovocitos vitelogenados o sin vitelo en proceso de atresia, pero con proporción no mayoritaria o frecuencia menor al 50\% (Fig. 5C).

\section{Desovante (IV)}

Son considerados como desovantes aquellos individuos que están en pleno desove o han desovado recientemente.

Características macroscópicas: Ovarios de gran tamaño y turgentes cuando están en pleno desove o previos a éste. Los ovocitos hidratados se pueden observar a simple vista. Los que ya han desovado presentan una coloración que va desde el verdoso hasta el ámbar. Cuando están parcialmente desovados son un tanto flácidos. En este estadio, es común apreciar una sectorización de los ovocitos hidratados en la gónada, en relación al resto de ellos de distribución más homogénea (Fig. 6 А y $6 \mathrm{~B})$.

Características microscópicas: En el caso de las hembras que se encuentran en pleno desove, la característica más resaltante es la presencia de los ovocitos hidratados y/o folículos post-ovulatorios. Si tienen folículos post-ovulatorios, éstos se visualizan de diferentes formas dependiendo del grado de degeneración en el que se encuentren. Estas características microscópicas que tipifican a ovarios desovantes, afortunadamente, para esta especie son de fácil identificación (Fig. 6C).

\section{Recuperación (V)}

Características macroscópicas: Este tipo de ovarios son poco frecuentes y se caracterizan por ser flácidos o no turgentes. Los ovocitos atrésicos visibles se observan a manera de grumos amarillos claros o puntos rojos u oscuros en la superficie ovárica. El ovario suele adquirir también este color (Fig. 7A).

Características microscópicas: El tejido ovárico presenta ovocitos atrésicos (atresia $\alpha$ ) en frecuencia igual o mayor al 50\%. También puede observarse restos de ovocitos vitelogenados o no vitelogenados en proceso de atresia en diferentes grados de degeneración $(\alpha, \beta, \mathrm{o} \gamma)$. Los ovocitos inmaduros y/o previtelogenados son los que prevalecen. Es perfectamente visible gran vascularización que le da el aspecto sanguinolento a este estadio (Fig. 7B).

\section{Escala de madurez gonadal de machos:}

\section{Virginal (0)}

Este estadio agrupa a los individuos machos que nunca han madurado gonadalmente.

Características macroscópicas: El testículo es pequeño, delgado y poco consistente, es de tonalidad rosada o rojiza, de apariencia translúcida, y se puede observar poco o nulo desarrollo vascular (Fig. 8A y 8B).

Características microscópicas: Pueden observarse zonas de crecimiento con la presencia de espermatogonias, donde igualmente se pueden encontrar algunos espermatocitos. (Fig. 8C).

\section{Reposo (I)}

Este estadio corresponde a individuos que han madurado gonadalmente alguna vez a lo largo de su vida, pero que se encuentran por el momento en una etapa de inactividad gonadal.

Características macroscópicas: Testículo de tamaño mediano, poco denso al tacto, de tonalidad anaranjada rojiza con algunas zonas claras y se puede observar un desarrollo vascular moderado (Fig. 9A y 9B).

Características microscópicas: Se observan los túbulos seminíferos con la presencia de espermatogonias y pueden observarse además algunos espermatocitos, con presencia de tejido conjuntivo que ocupan el espacio entre los túbulos seminíferos. Pared del testículo gruesa (Fig. 9C).

\section{En maduración (II)}

Estadio que agrupa a los individuos que dan inicio a la maduración testicular.

Características macroscópicas: Testículo de tamaño mediano a grande, que consiste en algunas zonas de color, desde blanco humo con presencia o no de algunas zonas rojizas hasta blanco cremoso y se puede observar desarrollo vascular moderado (Fig. 10A).

Características microscópicas: Túbulos seminíferos con presencia de espermatogonias, espermatocitos y espermátides, los cuales se observan de tamaño homogéneo. En ciertos sectores pueden o no observarse la presencia de algunos espermatozoides (Fig. 10-B).

\section{Maduro (III)}

Características macroscópicas: Gónada de gran tamaño, color blanquecino a blanco lechoso, turgente y con desarrollo vascular evidente (Fig. 11A y 11B).

Características microscópicas: Túbulos seminíferos con presencia de espermatogonias, espermatocitos y espermátides. En el lumen de los túbulos se observa la presencia de espermatozoides. El tubo colector común se presenta completamente lleno y de aspecto turgente (Fig. 11C).

\section{Expulsante (IV)}

Características macroscópicas: En su etapa inicial, los testículos tienen un tamaño máximo, muy turgentes, de color blanco lechoso y el líquido espermático se expele fácilmente con una suave presión. En su etapa avanzada, van disminuyendo de tamaño y disminuye la turgencia. De color blanquecino, y pueden presentar algunos sectores con tonalidades rosadas o rojizas (Fig. 12A).

Características microscópicas: Túbulos seminíferos con poca presencia de espermatogonias y espermatocitos, dominados por espermátides y espermatozoides que rellenan los túbulos. Es posible observar otras zonas vacías producto de la expulsión (Fig. 12B).

\section{Post-explusante (V)}

Características macroscópicas: Los testículos disminuyen de tamaño y su coloración varía a tonos más rojizos, debido al incremento de la irrigación sanguínea por efecto del evento de evacuación del líquido espermático. Son de consistencia flácida, debido al término del proceso de evacuación (Figs. 13A y 13B).

Características microscópicas: Se puede observar espermatogonias, espermatocitos y restos de espermatozoides en los túbulos seminíferos. En una etapa avanzada se pueden observar túbulos casi vacíos con material residual (Fig. 13C).

\section{Discusión}

La escala de madurez propuesta en este trabajo contempla las recomendaciones sugeridas por el "Grupo de trabajo sobre 


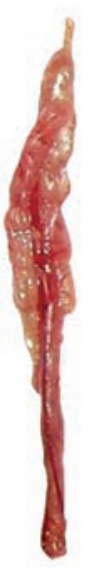

(A)

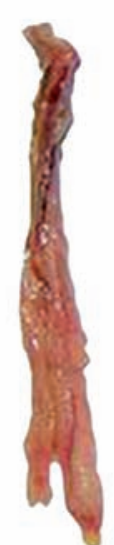

(B)

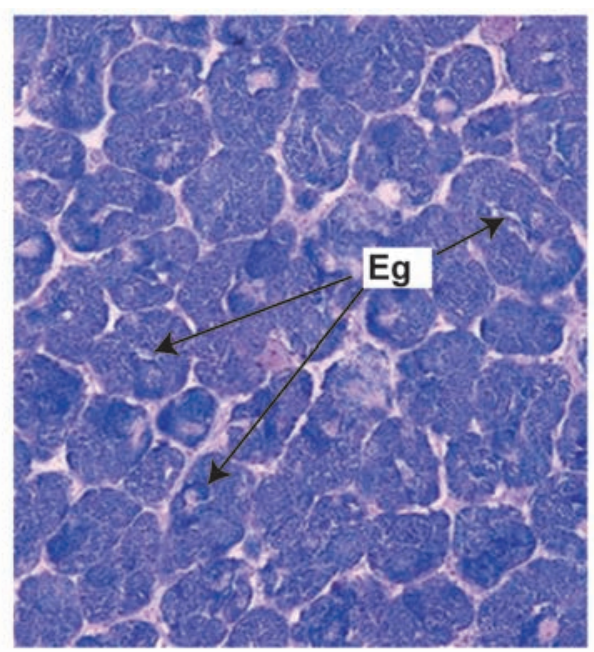

(C)

Figura 8. A y B) Fotografías de testículos de ejemplares de $25 \mathrm{~cm}$ y $26 \mathrm{~cm}$ de longitud total y C) Microfotografía (100X) de testículo de jurel Trachurus murphyi en estadio 0 (virginal). Eg: Espermatogonia.

Figure 8. A and B) Photographs of testicles of individuals of $25 \mathrm{~cm}$ and $26 \mathrm{~cm}$ TL and C) Photomicrograph (100X) of a testicle of Jack mackerel Trachurus murphyi in stage 0 (virgin). Eg: Spermatogonia.

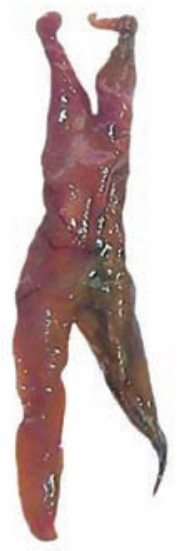

(A)

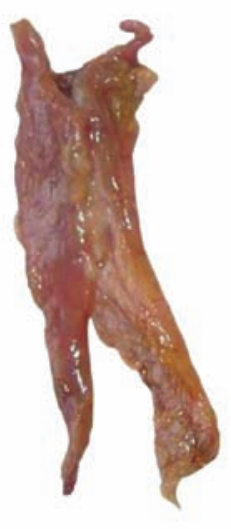

(B)

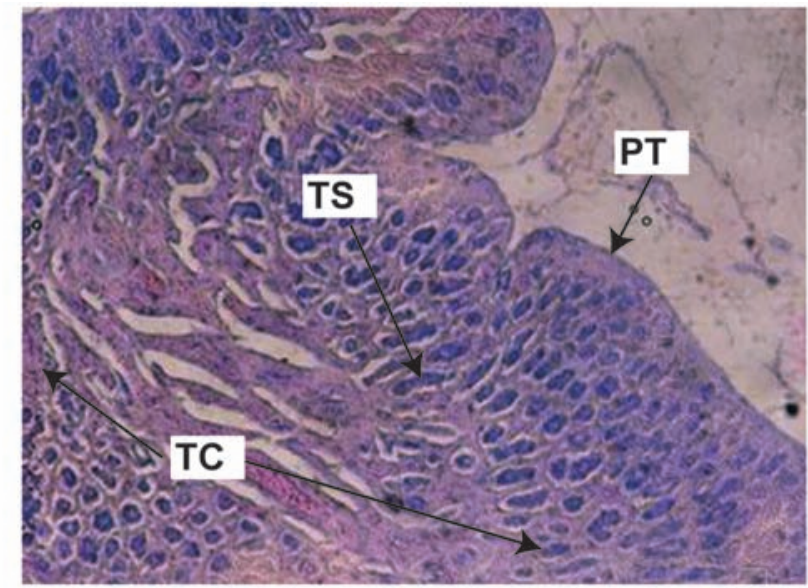

(C)

Figura 9. A y B) Fotografías de testículos de ejemplares $40 \mathrm{~cm}$ y $42 \mathrm{~cm}$ de longitud total y C) Microfotografía (40X) de testículo de jurel Trachurus murphyi en estadio I (reposo). TS: Túbulo seminífero, TC: Tejido conjuntivo, PT: Pared testicular.

Figure 9. A and B) Photographs of testicles of individuals of $40 \mathrm{~cm}$ and $42 \mathrm{~cm}$ TL and C) Photomicrograph (40X) testicles of Jack mackerel Trachurus murphyi in stage I (resting). TS: Seminiferous tubule, TC: Connective tissue, PT: Testicular Wall.

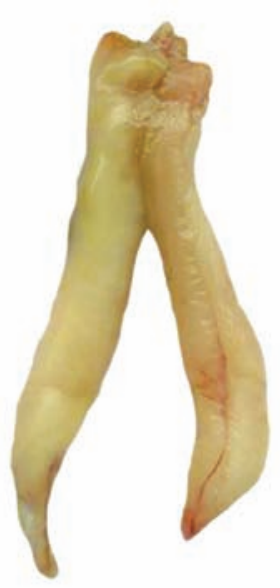

(A)

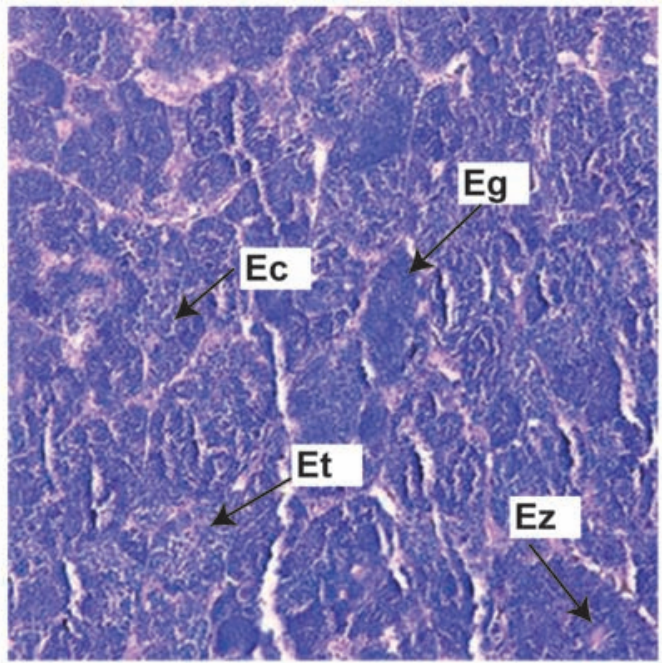

(B)

Figura 10. A) Fotografía de testículo de ejemplar de $29 \mathrm{~cm}$ de longitud total y B) Microfotografía (100X) de testículo de jurel Trachurus murphyi en estadio II (en maduración). Eg: Espermatogonia, Ec: Espermatocito, Et: Espermátide, Ez: Espermatozoide.

Figure 10. A) Photograph of a testicle of an individual of $29 \mathrm{~cm}$ total length and B) Photomicrograph (100X) of a testicle of Jack mackerel Trachurus murphyi in stage II (maturing). Eg: Spermatogonia, Ec: Spermatocytes, Et: Spermatid; Ez: Sperm. 

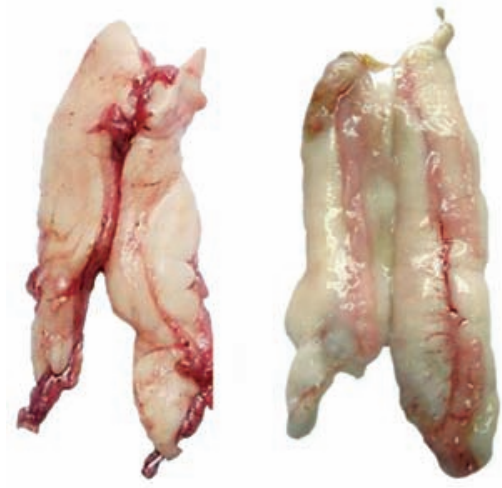

(A)
(B)

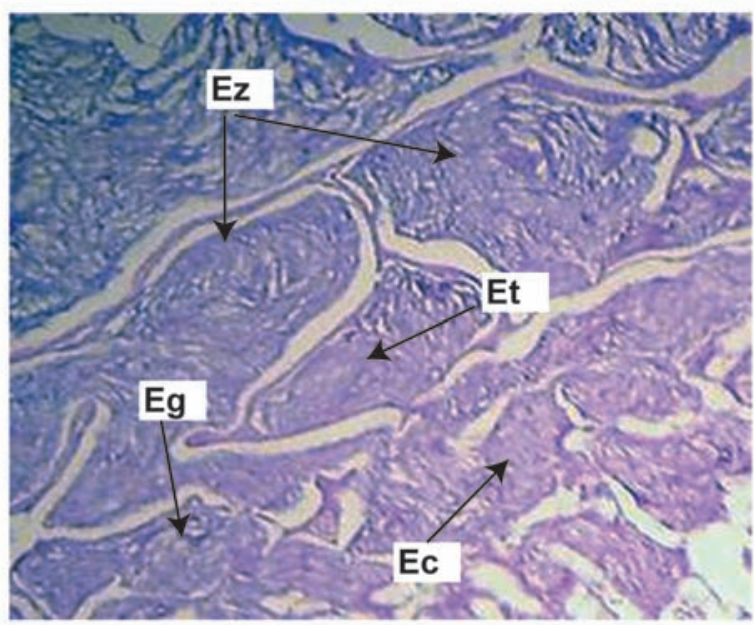

(C)

Figura 11. A y B) Fotografías de testículos de ejemplar de $34 \mathrm{~cm}$ y $39 \mathrm{~cm}$ de longitud total y C) Microfotografía (100x) de testículo de jurel Trachurus murphyi en estadio III (maduro). Eg: Espermatogonia, Ec: Espermatocito, Et: Espermátides, Ez: Espermatozoide.

Figure 11. A and B) Photographs of testicles of individuals of $34 \mathrm{~cm}$ and $39 \mathrm{~cm}$ TL and C) Photomicrograph (100x) of testicles of Jack mackerel Trachurus murphyi in stage III (mature). Eg: Spermatogonia, Ec: Spermatocytes, Et: Spermatids, Ez: Sperm.

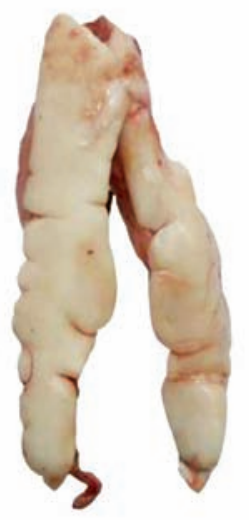

(A)

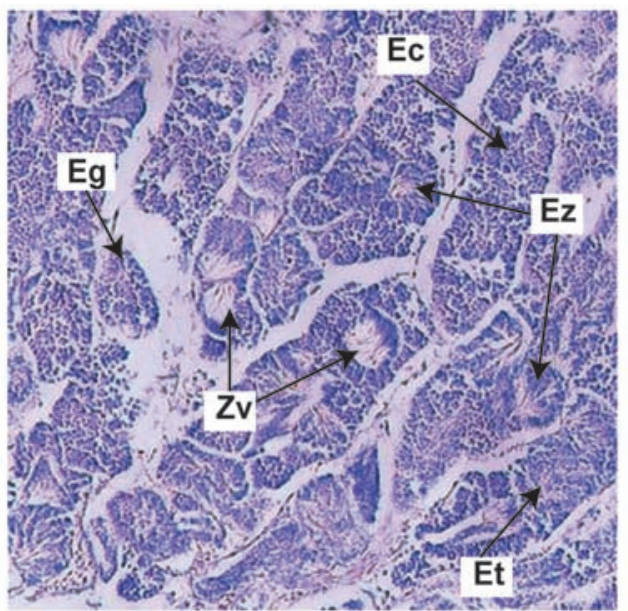

(B)

Figura 12. A) Fotografía de testículo de ejemplar de $34 \mathrm{~cm}$ de longitud total y B) Microfotografía (100X) de testículo de jurel Trachurus murphyi en estadio IV (expulsante). Eg: Espermatogonia, Ec: Espermatocito, Et: Espermátide, Ez: Espermatozoide, ZV: Zonas vacías producto de la expulsión.

Figure 12. A) Photograph of a testicle of an individual of $34 \mathrm{~cm} \mathrm{TL}$ and B) Photomicrograph (100X) of a testicle of Jack mackerel Trachurus murphyi in stage IV (spermiation). Eg: Spermatogonia, Ec: Spermatocytes, Et: Spermatid; Ez: Sperm, ZV: Empty zones.

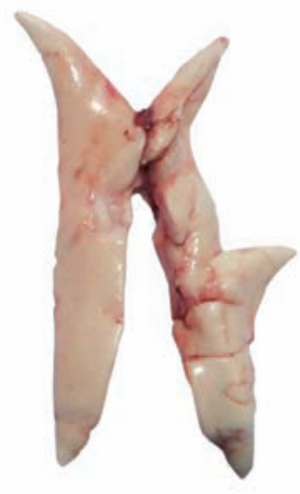

(A)

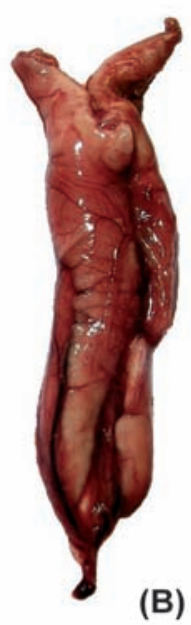

(B)

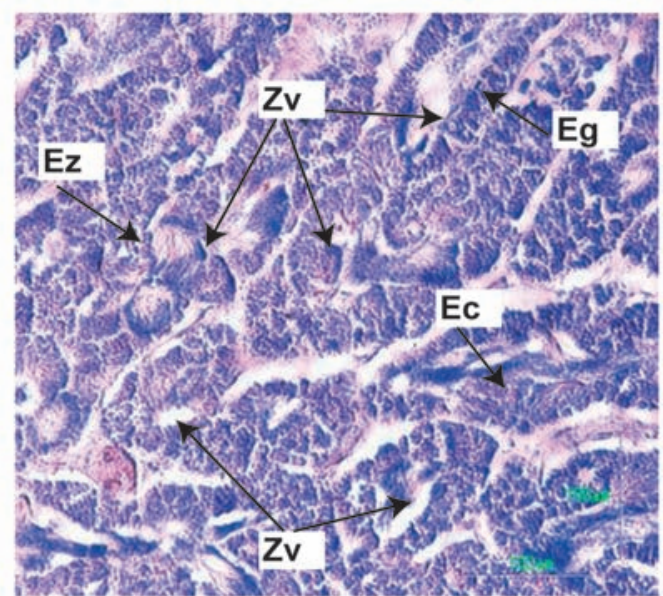

(C)

Figura 13. A y B) Fotografías de testículos de ejemplares de $35 \mathrm{~cm}$ (etapa inicial) y $39 \mathrm{~cm}$ (etapa avanzada) de longitud total y C) Microfotografía (100X) de testículo de jurel Trachurus murphyi en estadio V (post-explulsante). Eg: Espermatogonia, Ec: Espermatocito, Ez: Espermatozoide, ZV: Zonas vacías producto de la expulsión.

Figure 13. A and B) Photographs of testicles of individuals of $35 \mathrm{~cm}$ (initial stage) and $39 \mathrm{~cm}$ (advanced stage) TL and C) Photomicrograph (100X) testicle of Jack mackerel Trachurus murphyi in stage V (post-spermiation). Eg: Spermatogonia, Ec: Spermatocytes, Ez: Sperm, ZV: Empty zones. 
enfoques modernos para evaluar la madurez y la fecundidad de los peces y calamares de aguas cálidas y frias" (Kjesbu et al. 2003), y tiene base en observaciones microscópicas que permiten plantear una escala de seis estadios, incluyendo a los individuos virginales catalogados como estadio 0 . El estadio 0 permite discriminar con facilidad a los individuos virginales y estimar los estadios de madurez sólo con la fracción adulta. Al mismo tiempo, hace posible obtener estructuras de madurez gonadal de la población más claras, con las que se pueden estimar proporciones de estadios de madurez por tallas, que son usadas para construir las ojivas de madurez.

Las características microscópicas de los ovarios denotan la tipificación de desove parcial que ya fue observado por Dioses (1988) y confirmado por Perea et al. (2003). Este hecho también ha sido observado en otras especies de jurel como Trachurus trachurus (Arruda 1984, Karlou-Riga \& Economidis 1997). En nuestro trabajo, las características de los tipos de ovocitos de jurel T. murphyi observados también coinciden con lo reportado por Dioses (1998), así como para otras especies del mismo género (Karlou-Riga \& Economidis 1997).

Cabe mencionar que para muchas especies no es posible distinguir a simple vista, las hembras inmaduras de las que se encuentran en "reposo" (Trippel \& Morgan 1997, SaboridoRey \& Junquera 1998, Domínguez-Petit 2007). Sin embargo, las gónadas del jurel T. murphyi tienen un mayor volumen, y por ello es mucho más fácil discriminar ambos estadios sin la necesidad de hacer cortes histológicos como lo sugieren Ferreri et al. (2009), que elaboraron la escala de madurez de la anchoveta Engraulis encrasicolus y recomendaron realizar cortes histológicos de las gónadas de los individuos durante los picos de desove, para poder diferenciar las hembras virginales de las que ya habían tenido desoves, y que se encontraban inactivas. Esta recomendación se realizaba para evitar que se confundan y clasifiquen erróneamente los estadios virginal e inactivo, lo que introduciría errores al estimar la proporción de individuos maduros de la población, sobre todo si parte de las hembras en reposo que ya habían contribuido a la biomasa reproductora son consideradas inmaduras.

El estadio en recuperación puede ser considerado como un estadio "confuso" por algunos autores (Hunter \& Macewicz 2003), pero en el caso del jurel T. murphyi este estadio es reconocible a simple vista por el color, pérdida de turgencia y disminución del volumen del ovario. Por otro lado, al igual que lo observado en los ovarios de merluza, debido a su dimensión, los ovocitos atrésicos de jurel T. murphyi se reconocen a simple vista al hacer un corte transversal del ovario, observándose como grumos que van desde el color amarillo claro hasta el rojizo. Se destaca que los niveles de atresia observados en jurel T. murphyi, al igual que en T. trachurus (Gordo et al. 2008) y en merluza Merluccius gayi peruanus, son mayores que los niveles de atresia observados en ovarios de anchoveta Engraulis ringens.

En conclusión, en el presente trabajo han sido descritas las fases de madurez gonadal del jurel T. murphyi basadas en características que pueden diferenciarse a simple vista y validadas con exámenes histológicos. Esta escala permitirá la estimación rápida de la fase de madurez de la población. Por otro lado, es recomendable que el uso de esta escala sea intercalibrada entre los usuarios de diferentes zonas, así como otros países, para estandarizar su uso, lo que conllevaría a disminuir la subjeti- vidad en la catalogación y el sesgo en los resultados (Gerritsen $\&$ McGrath 2006, Ferreri et al.2009).

\section{Literatura citada}

Abascal F., C. Megina \& A. Medina. 2004. Testicular development in migrant and spawning bluefin tuna Thunnus thynnus (L.) from the eastern Atlantic and Mediterraneam. Fish. Bull. 102: 407-417.

Andrianov D.D. 1987. Sobre la biología reproductiva del jurel peruano. En: Biología y pesca del jurel peruano. VNIRO, Moscú: 20-34. (En ruso).

Aracena O., R. Alarcón, S. Collado, I. Lépez, \& D. Arriagada. 1998. Aspectos reproductivos del jurel (Trachurus symmetricus murphyi) de la pesquería de cerco de la Octava Región, Chile, 1994-1995. In: Arcos, D. (ed.), Biología y ecología del jurel en aguas chilenas, Instituto de Investigación Pesquera, Talcahuano, Chile, p. 101-114.

Arruda L.M. I984. Sexual maturation and growth of Trachurus trachurus (L.) along the Portuguese coast. Inv. Pesq. 48(3): 419-430.

Costa A.M. 2001. Contribuição para o estudo da fecundidade temporal e espacial do carapau (Trachurus trachurus L.) na costa Portuguesa. Tese para acesso à categoria de Investigador Auxiliar - IPIMAR, 180 pp.

Costa A.M. \& M.F. Borges. 1996. Total fecundity and atresia of horse mackerel (Trachurus trachurus L.). ICES, C. M.1996/ H: 26.

Cubillos L. (Ed.) 2003. Informe final, proyecto FIP 2001-12, condición biológica del jurel en alta mar, año 2001. Fondo de Investigación Pesquera - Instituto de Investigación Pesquera, Chile, Octava Región: 212 pp. <http://www2.udec.cl/ - coyarzun/inffinalFIP2001-12.pdf $>$ [acceso 16/08/2013]

Dadzie S. \& F. Abou-Seedo. 2004. Testicular structure and spawning cycle in the silvery croaker, Otolithes ruber (Perciformes: Scianidae) in the Kuwaiti waters of the Arabian Gulf. Ichthyol. Res. 51:263-268 .

Dioses T., V. Alarcón, M.H. Nakama \& A. Echeverría. 1989. Desarrollo ovocitario, fecundidad parcial y distribución vertical de los cardúmenes en desove del jurel Trachurus murphyi (N). En: R. Jordán, R. Kelly, O. Mora, A. de Vildoso \& N. Henríquez (eds.). Memorias del Simposio internacional sobre recursos vivos y pesquerías en el Pacífico sudeste. Rev. Com. Perm. Pacífico Sur. Número especial: 287-294.

Domínguez-Petit R. 2007. Study of Reproductive Potential of Merluccius Merluccius in the Galician Shelf. Doctoral Thesis. University of Vigo, Vigo, Spain: 253 pp.

Ferreri R., G. Basilone, M. D’Elia, A. Traina, F. Saborido-Rey \& S. Mazzola. 2009. Validation of macroscopic maturity stages according to microscopic histological examination for european anchovy. Marine Ecology 30: 181-187. doi:10.1111/j.1439-0485.2009.00312.x.

Gerritsen H.D. \& D. McGrath. 2006. Variability in the assignment of maturity stages of plaice (Pleuronectes platessa L.) and whiting (Merlangius merlangus L.) using macroscopic maturity criteria. Fisheries Research 77:72-77.

Gonçalves P., E. Cunha \& A. Costa. 2004. Escala de maturação microscópica e macroscópica das gónadas femininas de pescada (Merluccius merluccius). Relat. Cient. Téc. IPIMAR, Série digital (http:// ipimariniap.ipimar.pt) $21.27 \mathrm{pp}$.

Gordo L.S., A. Costa, P. Abaunza et al.2008. Determinate versus indeterminate fecundity in horse mackerel. Fisheries Research 89:181-185.

Gluyas-Millán M.G. 1994. Reproducción, edad y talla de primera madurez de la macarela Scomber japonicus (Houttuyn 1872) en Bahía Vizcaíno. Ciencias Marinas 20(3): 409-419.

Holden M.J. \& D.F.S. Raitt. 1975. Manual de ciencia pesquera. Parte 2: Métodos para investigar los recursos y su aplicación. Doc. Téc. FAO Pesca (115). Rev 1: 211 p.

Humason G.L. 1979. Animal tissue techniques. Fourth edition. W. H. Freeman Co. San Francisco. 661 pp.

Hunter J. \& S. Goldberg. 1980. Spawning incidence and batch fecundity in northern anchovy, Engraulis mordax. Fish. Bull. 77: 641-652.

Hunter J.R. \& B.J. Macewicz. I985. Measurement of spawning frequency in multiple spawning fish. In R. Lasker (Ed.). An Egg Production Method for Estimating Spawning Biomass of Pelagic Fish: Application to the Northern Anchovy, Engraulis mordax. NOAA Technical Report NMFS 36. Pp. 79-94

Hunter J.R. \& B.J. Macewicz. 2003. Improving the accuracy and precision of reproductive information used in fisheries. In: O.S. Kjesbu, J.R. Hunter and P. Witthames (Eds.), Report of the Working Group on Modern Approaches to Assess Maturity and Fecundity of Warm- and Coldwater Fish and Squids. Fisken og 12. 
Johansen A.C. 1924. On the summer and autumn spawning herring of the North Sea. Meddelelser fra Kommissionen for Havundersøgelser. Serie Fiskeri, Bind VII, Nr.5, 119 pp., 15 figs. København.

Karlou-Riga C. \& P.S. Economidis. 1997. Spawning frequency and batch fecundity of horse mackerel, Trachurus trachurus (L.), in the Saronikos Gulf (Greece). J. Appl. Ichthyol. 13:97-104.

Kjesbu O., R. Hunter \& P. Witthames (Ed.). 2003. Plenary document. In: Report of the working group on modern approaches to assess maturity and fecundity of warm- and cold-water fish and squids. Fisken og havet 2003-12. http://brage.bibsys.no/imr/handle/ URN:NBN:nobibsys_brage_3594. [acceso: 18/07/2013]
Pérez N. \& I. Figueiredo. 1992. First approach to the study of atresia in the ovary of sardine Sardine pilchardus (Walb.). Bol. Inst. Esp. Oceanogr. 8(1):191-199.

Perea A., J. Mori, B. Buitrón \& J. Sánchez. 2013. Aspectos reproductivos del jurel Trachurus murphyi (Nichols 1920) en el Perú. En: Csirke J., R. Guevara-Carrasco, M. Espino (Eds.). Ecología, pesquería y conservación del jurel (Trachurus murphyi) en el Perú. Rev. peru. biol. número especial 20(1): 029 - 034

Saborido-Rey F. \& S. Junquera. I 998. Histological assessment of variations in sexual maturity of cod (Gadus morhua L.) at the Flemish Cap (north-west Atlantic). ICES Journal of Marine Science 55:515-521.

Trippel E.A. \& M.J. Morgan. I997. Skewed sex ratios in spawning shoals of Atlantic cod (Gadus morhua). Oceanographic Literature Review $44,511 \mathrm{pp}$ 